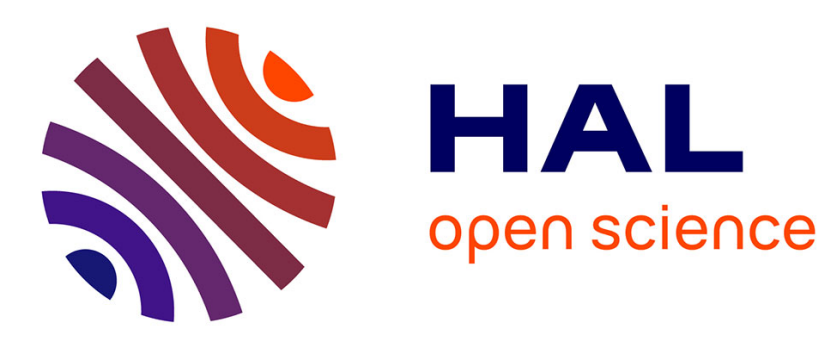

\title{
A simple method for measuring power, force, velocity properties, and mechanical effectiveness in sprint running
}

Pierre Samozino, Giuseppe Rabita, Sylvain Dorel, Jean Slawinski, Nicolas

Peyrot, Eduardo Saez de Villareal, Jean-Benoît Morin

\section{To cite this version:}

Pierre Samozino, Giuseppe Rabita, Sylvain Dorel, Jean Slawinski, Nicolas Peyrot, et al.. A simple method for measuring power, force, velocity properties, and mechanical effectiveness in sprint running. Scandinavian Journal of Medicine and Science in Sports, 2015, 26, pp.648 - 658. 10.1111/sms.12490 . hal-01389134

\section{HAL Id: hal-01389134 \\ https://hal.univ-reunion.fr/hal-01389134}

Submitted on 28 Oct 2016

HAL is a multi-disciplinary open access archive for the deposit and dissemination of scientific research documents, whether they are published or not. The documents may come from teaching and research institutions in France or abroad, or from public or private research centers.
L'archive ouverte pluridisciplinaire HAL, est destinée au dépôt et à la diffusion de documents scientifiques de niveau recherche, publiés ou non, émanant des établissements d'enseignement et de recherche français ou étrangers, des laboratoires publics ou privés. 


\title{
A simple method for measuring power, force, velocity properties, and mechanical effectiveness in sprint running
}

\author{
P. Samozino ${ }^{1}$, G. Rabita ${ }^{2}$, S. Dorel ${ }^{3}$, J. Slawinski ${ }^{4}$, N. Peyrot ${ }^{5}$, E. Saez de Villarreal ${ }^{6}$, J.-B. Morin $^{7}$ \\ ${ }^{1}$ Laboratory of Exercise Physiology (EA4338), University Savoie Mont Blanc, Le Bourget du Lac, France, ${ }^{2}$ Research Department, \\ Laboratory Sport, Expertise and Performance, French Institute of Sport (INSEP), Paris, France, ${ }^{3}$ Laboratory "Motricite, Interactions, \\ Performance" (EA 4334), University of Nantes, Nantes, France, ${ }^{4}$ CeSERM - EA 2931, UFRSTAPS, Université de Paris Ouest \\ Nanterre la Défense, Paris, France, ${ }^{5}$ Laboratory IRISSE (EA4075), University of La Réunion, Le Tampon, La Réunion, France, \\ ${ }^{6}$ Laboratory of Human Performance, Department of Sports, University Pablo de Olavide, Seville, Spain, ${ }^{7}$ Laboratory of Human \\ Motricity, Education Sport and Health (EA6312), University of Nice Sophia Antipolis, Nice, France \\ Corresponding author: Pierre Samozino, PhD, Laboratoire de Physiologie de l'Exercice, Université de Savoie Mont-Blanc, UFR \\ CISM - Technolac, 73376 Le Bourget du Lac, France. Tel: +33 4797581 77, Fax: +33 4797581 48, E-mail: \\ pierre.samozino@univ-savoie.fr
}

This study aimed to validate a simple field method for determining force- and power-velocity relationships and mechanical effectiveness of force application during sprint running. The proposed method, based on an inverse dynamic approach applied to the body center of mass, estimates the step-averaged ground reaction forces in runner's sagittal plane of motion during overground sprint acceleration from only anthropometric and spatiotemporal data. Force- and power-velocity relationships, the associated variables, and mechanical effectiveness were determined (a) on nine sprinters using both the proposed method and force plate measurements and (b) on six other sprinters using the proposed method during

Sprint running is a key factor of performance in many sport activities, not only to reach the highest top velocity but also, and most importantly, to cover a given distance in the shortest time possible, be it in track-and-field events or in team sports. This ability implies large forward acceleration, which has been related to the capacity to produce and apply high amounts of power output in the horizontal direction onto the ground, i.e., high amounts of horizontal external force at various velocities over sprint acceleration (Jaskolska et al., 1999b; Morin et al., 2011a, 2012; Rabita et al., 2015).

The overall mechanical capability to produce horizontal external force during sprint running is well described by the inverse linear force-velocity $(\mathrm{F}-\mathrm{v})$ and the parabolic power-velocity $(\mathrm{P}-\mathrm{v})$ relationships (Jaskolska et al., 1999a, b; Morin et al., 2010, 2011a, 2012; Rabita et al., 2015). Indeed, although the $\mathrm{F}-\mathrm{v}$ relationships obtained on isolated muscles or mono-articular movements are described by a hyperbolic equation (Hill, 1938; Thorstensson et al., 1976), linear relationships were consistently obtained for multijoint lower limb movements such as pedaling, squat, leg press, or sprint several consecutive trials to assess the inter-trial reliability. The low bias $(<5 \%)$ and narrow limits of agreement between both methods for maximal horizontal force $(638 \pm 84 \mathrm{~N})$, velocity $(10.5 \pm 0.74 \mathrm{~m} / \mathrm{s})$, and power output $(1680 \pm 280 \mathrm{~W})$; for the slope of the force-velocity relationships; and for the mechanical effectiveness of force application showed high concurrent validity of the proposed method. The low standard errors of measurements between trials $(<5 \%)$ highlighted the high reliability of the method. These findings support the validity of the proposed simple method, convenient for field use, to determine power, force, velocity properties, and mechanical effectiveness in sprint running.

running movements (e.g., Yamauchi \& Ishii, 2007; Dorel et al., 2010; Morin et al., 2010; Bobbert, 2012; Samozino et al., 2012; Rabita et al., 2015). These relationships characterize the external mechanical limits of the entire neuromuscular system during specific multijoint movements and are well summarized through the theoretical maximal force $\left(F_{0}\right)$ and velocity $\left(v_{0}\right)$ this system can develop, and the associated maximal power output $\left(P_{\max }\right)$. Moreover, the slope of the $\mathrm{F}-\mathrm{v}$ relationship determines the $\mathrm{F}-\mathrm{v}$ mechanical profile $\left(S_{\mathrm{FV}}\right)$, i.e., the individual ratio between force and velocity qualities.

These mechanical properties obtained from multijoint $\mathrm{F}-\mathrm{V}$ and $\mathrm{P}-\mathrm{v}$ relationships are a complex integration of different mechanisms involved in the total external force produced during one (for acyclic movements) or several consecutive (for cyclic movements) limb extensions. They encompass individual muscle mechanical properties, morphological factors, neural mechanisms, and segmental dynamics (Cormie et al., 2010a, b, 2011; Bobbert, 2012). Furthermore, because sprint running is a dynamic movement mainly requiring force production in two dimensions (in contrast to squat or leg press 
exercises), the $\mathrm{F}-\mathrm{v}$ and $\mathrm{P}-\mathrm{v}$ relationships during running propulsion also integrate the ability to apply the external force effectively (i.e., horizontally in the antero-posterior direction) onto the ground (Morin et al., 2011a, b, 2012; Rabita et al., 2015). This concept of mechanical effectiveness of force application has been previously proposed in sprint pedaling considering tangential and normal force components of the force applied onto the pedals (Dorel et al., 2010). The technical ability of force application (or mechanical effectiveness) during sprint running has been quantified at each step by the ratio $(R F)$ of the net horizontal and resultant ground reaction forces (GRF), and over the entire acceleration phase by the rate $\left(D_{\mathrm{RF}}\right)$ of linear decrease in $R F$ as velocity increases. $D_{\mathrm{RF}}$, which is independent from the amount of total force applied (i.e., physical capabilities), describes the runner's ability to maintain a forward horizontal orientation of the resultant GRF vector despite increasing speed (Morin et al., 2011a, b; Morin et al., 2012; Rabita et al., 2015; see Methods section). So, F-v and $\mathrm{P}-\mathrm{v}$ relationships provide a macroscopic and integrative view of the $\mathrm{P}-\mathrm{F}-\mathrm{v}$ mechanical profile of an athlete in the specific sprint running task.

Therefore, determining individual $\mathrm{F}-\mathrm{v}$ and $\mathrm{P}-\mathrm{v}$ relationships and mechanical effectiveness during sprint propulsion is of great interest for coaches, sport practitioners, or physiotherapists. Indeed, sprint performance is highly correlated to $P_{\max }$, be it quantified during sprint running or other movements such as vertical jump or sprint cycling (e.g., Cronin \& Sleivert, 2005; Morin et al., 2012; Rabita et al., 2015). In addition to this power output capability, the $\mathrm{F}-\mathrm{v}$ mechanical profile (characterized by the slope of the $\mathrm{F}-\mathrm{v}$ relationship) has recently been shown to influence maximal jumping performances, independently from the effect of $P_{\max }$, with the existence of an individual optimal $\mathrm{F}-\mathrm{v}$ profile characterizing the best balance, for a given subject, between force and velocity qualities to maximize performances (Samozino et al., 2012, 2014). These results suggest that the $\mathrm{F}-\mathrm{v}$ mechanical profile in sprint running, which shows high inter-individual differences (Jaskolska et al., 1999b; Morin et al., 2010), can also be interesting to consider and adjust by individualized training loads and exercises. Finally, recent studies showed that sprint performances (6-s sprints, 100-m events, repeated sprints) are related more to the effectiveness of force application to the ground than to the total force developed by lower limbs (Morin et al., 2011a, 2012; Rabita et al., 2015). So, quantifying individually the mechanical effectiveness can help distinguish the physical and technical origins of inter- or intra-individual differences in both $\mathrm{P}-\mathrm{F}-\mathrm{V}$ mechanical profiles and sprint performances, which can be useful to more appropriately orient the training process toward the specific mechanical qualities to develop.

To date, such evaluations require to measure horizontal antero-posterior and vertical GRF components and forward horizontal velocity during an entire sprint acceleration $(\sim 30-60 \mathrm{~m})$. Sprint $\mathrm{F}-\mathrm{v}$ and $\mathrm{P}-\mathrm{v}$ relationships have hitherto been obtained using specific instrumented treadmills on which subjects accelerate the belt themselves by the action of their lower limbs, while their waist is tethered backward to a fixed point (e.g., Jaskolska et al., 1999a, b; Chelly \& Denis, 2001; Morin et al., 2010). Despite the high accuracy of these methods, their main limitation is the treadmill condition that does not exactly reproduce natural overground sprint running movement due to waist attachment, a belt narrower than a typical track lane, the impossibility to use starting block, and the need to set a default torque to, only partly, compensate for the friction of the treadmill belt bed (Morin et al., 2010; Morin \& Seve, 2011). Because, to date, no 30- to 60-m long force plate systems exist, GRFs over an entire sprint acceleration phase were very recently determined in elite sprinters using data from several sprints measured on a $6.60-\mathrm{m}$ long force plate system, which allowed, for the first time, to provide the data to entirely characterize the mechanics of overground sprint acceleration (Rabita et al., 2015). Sport practitioners do not have easy access to such rare and expensive devices, and often do not have the tech-nical expertise to process the raw force data measured. In the best cases, this forces athletes to report to a labora-tory, which explains that, although very accurate and potentially useful for training purposes, this kind of evaluation is almost never performed. In addition, sport scientists investigating sprint mechanics and perfor-mance usually assess, at best, only very few steps of a sprint because of this technical limitation (e.g., Lockie et al., 2013; Kawamori et al., 2014). A simple method for determining $\mathrm{F}-\mathrm{v}$ and $\mathrm{P}-\mathrm{v}$ relationships and force appli-cation effectiveness during sprint running in overground realistic conditions could therefore be very interesting to generalize such evaluations for training or scientific purposes.

Mechanics and energetics of sprint running have been approached by various kinds of mathematical models that aimed at describing sprint performance from the balance between the mechanical energy demand of sprint running acceleration and the energy release capacity of the aerobic and anaerobic metabolism (e.g., Furusawa et al., 1927; van Ingen Schenau et al., 1991; Arsac \& Locatelli, 2002; Helene \& Yamashita, 2010). Based on the mechanical analyses used in these models, an inverse dynamic approach applied to the runner body center of mass $(\mathrm{CM})$ could give valid estimation of GRF during sprint running acceleration from simple kinematic data, as recently proposed by di Prampero et al. (2015), but never compared with force plate measurements. This could then be used to obtain the aforementioned sprint mechanical properties without force platform system in typical field conditions of practice.

The aim of this study was to propose and validate a simple field method based on an inverse dynamic 
approach applied to the runner body CM during sprint running acceleration for (a) estimating the GRFs in the sagittal plane of motion from only anthropometric and spatiotemporal data and (b) determining the associated $\mathrm{F}-\mathrm{v}$ and $\mathrm{P}-\mathrm{v}$ relationships and effectiveness of force application. The concurrent validity of this method was tested by comparison to reference force plate measurements.

\section{Biomechanical model used in the proposed method}

This section, devoted to present the biomechanical model on which the proposed simple method is based, is an analysis of kinematics and kinetics of the runner's body CM during sprint acceleration using a macroscopic inverse dynamic approach aiming to be the simplest possible (Furusawa et al., 1927; Helene \& Yamashita, 2010). All variables presented in this section are modeled over time, without considering intra-step changes, and thus correspond to step-averaged values (contact plus aerial times).

During a running maximal acceleration, horizontal velocity $\left(v_{\mathrm{H}}\right)$-time $(t)$ curve has long been shown to systematically follow a mono-exponential function for recreational to highly trained sprinters (e.g., Furusawa et al., 1927; Chelly \& Denis, 2001; di Prampero et al., 2005; Morin et al., 2006):

$$
v_{H}(t)=v_{H_{\max }} \cdot\left(1-e^{-t / \tau}\right)
$$

where $v_{H_{\max }}$ is the maximal velocity reached at the end of the acceleration and $\tau$ is the acceleration time constant. The horizontal position $\left(x_{\mathrm{H}}\right)$ and acceleration $\left(a_{\mathrm{H}}\right)$ of the body $\mathrm{CM}$ as a function of time during the acceleration phase can be expressed after integration and derivation of $v_{\mathrm{H}}(t)$ over time, respectively, as follows:

$$
\begin{gathered}
x_{\mathrm{H}}(t)=\int v_{\mathrm{H}}(t) d t=\int v_{\mathrm{H}_{\text {max }}} \cdot\left(1-e^{-\frac{t}{\tau}}\right) d t \\
x(t)=v_{\mathrm{H}_{\text {max }}} \cdot\left(t+\tau \cdot e^{-\frac{t}{\tau}}\right)-v_{\mathrm{H}_{\text {max }}} \cdot \tau \\
a_{\mathrm{H}}(t)=\frac{d v_{\mathrm{H}}(t)}{d t}=\frac{v_{\mathrm{H}_{\text {max }}} \cdot\left(1-e^{-\frac{t}{\tau}}\right)}{d t} \\
a_{\mathrm{H}}(t)=\left(\frac{v_{\text {max }}}{\tau}\right) \cdot e^{-\frac{t}{\tau}}
\end{gathered}
$$

Applying the fundamental laws of dynamics in the horizontal direction, the net horizontal antero-posterior GRF $\left(F_{\mathrm{H}}\right)$ applied to the body $\mathrm{CM}$ can be modeled over time as:

$$
F_{\mathrm{H}}(t)=m \cdot a_{\mathrm{H}}(t)+F_{\text {aero }}(t)
$$

where $m$ is the runner's body mass (in $\mathrm{kg}$ ) and $F_{\text {aero }}(t)$ is the aerodynamic drag to overcome during sprint running that is proportional to the square of the velocity of air relative to the runner:

$$
F_{\text {aero }}(t)=k \cdot\left(v_{\mathrm{H}}(t)-v_{\mathrm{w}}\right)^{2}
$$

where $v_{\mathrm{w}}$ is the wind velocity (if any) and $k$ is the runner's aerodynamic friction coefficient, which can be estimated as proposed by Arsac and Locatelli (2002) from values of air density $\left(\rho\right.$, in $\left.\mathrm{kg} / \mathrm{m}^{3}\right)$, frontal area of the runner $\left(A f\right.$, in $\left.\mathrm{m}^{2}\right)$, and drag coefficient $(C d=0.9$; van Ingen Schenau et al., 1991):

$$
k=0.5 \cdot \rho \cdot A f \cdot C d
$$

with

$$
\begin{gathered}
\rho=\rho_{0} \cdot \frac{P b}{760} \cdot \frac{273}{273+T^{\circ}} \\
A f=\left(0.2025 \cdot h^{0.725} \cdot m^{0.425}\right) \cdot 0.266
\end{gathered}
$$

where $\rho_{0}=1.293 \mathrm{~kg} / \mathrm{m}$ is the $\rho$ at 760 Torr and $273{ }^{\circ} \mathrm{K}$, $P b$ is the barometric pressure (in Torr), $T^{\circ}$ is the air temperature (in ${ }^{\circ} \mathrm{C}$ ), and $h$ is the runner's stature (in $\mathrm{m}$ ). The mean net horizontal antero-posterior power output applied to the body $\mathrm{CM}\left(P_{\mathrm{H}}\right.$ in W) can then be modeled at each instant as the product of $F_{\mathrm{H}}$ and $v_{\mathrm{H}}$.

In the vertical direction, the runner's body $\mathrm{CM}$ during the acceleration phase of a sprint goes up from the starting crouched position (be it with or without using starting blocks) to the standing running position, and then does not change from one complete step to another. Because the initial upward movement of the $\mathrm{CM}$ is overall smoothed through a relative long time/distance ( $\sim 30-40 \mathrm{~m}$; Cavagna et al., 1971; Slawinski et al., 2010), we can consider that it does not require any large vertical acceleration, and so that the mean net vertical acceleration of the $\mathrm{CM}$ over each step is quasi-null throughout the sprint acceleration phase. Consequently, applying the fundamental laws of dynamics in the vertical direction, the mean net vertical GRF $\left(F_{\mathrm{V}}\right)$ applied to the body $\mathrm{CM}$ over each complete step can be modeled over time as equal to body weight (di Prampero et al., 2015):

$$
F_{\mathrm{V}}(t)=m \cdot g
$$

where $g$ is the gravitational acceleration $\left(9.81 \mathrm{~m} / \mathrm{s}^{2}\right)$.

Morin et al. (2011a) proposed that the mechanical effectiveness of force application during running could be quantified over each support phase or step by the ratio ( $R F$ in \%, eqn. [12]) of $F_{\mathrm{H}}$ to the corresponding total resultant GRF $\left(F_{\text {Res, }}\right.$, in $\left.\mathrm{N}\right)$, and over the entire 
acceleration phase by the slope of the linear decrease in $R F$ when velocity increases $\left(D_{\mathrm{RF}}\right.$, in \%.s/m):

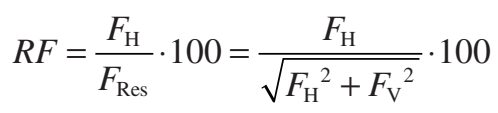

To date, $R F$ values have been computed from the second step (i.e., the first complete step) to the step at maximal speed (Morin et al., 2011a, b, 2012; Rabita et al., 2015). Because the starting block phase (push-off and following aerial time) lasts between 0.5 and $0.6 \mathrm{~s}$ (Slawinski et al., 2010; Rabita et al., 2015) and so occurs at an averaged time of $\sim 0.3 \mathrm{~s}, R F$ and $D_{\mathrm{RF}}$ can be reasonably computed from $F_{\mathrm{H}}$ and $F_{\mathrm{V}}$ values modeled for $t>0.3 \mathrm{~s}$.

The above-described biomechanical model makes possible to estimate GRFs in the sagittal plane of motion during one single sprint running acceleration from simple inputs: anthropometric (body mass and stature) and spatiotemporal (split times or instantaneous running velocity) data. This model can then be used as a simple method to determine the $\mathrm{F}-\mathrm{v}$ and $\mathrm{P}-\mathrm{v}$ relationships and the associated variables, as well as the mechanical effectiveness of force application parameters (see Methods section for details about the practical methodology associated to this computation method). Two different experimental protocols were conducted (a) to test the concurrent validity of the proposed computation method by comparison to force plate measurements and (b) to test its inter-trial reliability.

\section{Methods}

First protocol: concurrent validity compared with force plate measurements

\section{Subjects and protocol}

Nine elite or sub-elite sprinters (age: $23.9 \pm 3.4$ years; body mass: $76.4 \pm 7.1 \mathrm{~kg}$; height: $1.82 \pm 0.69 \mathrm{~m})$ gave their written informed consent to participate in this study, which was approved by the local ethical committee and in agreement with the Declaration of Helsinki. Their personal $100-\mathrm{m}$ official best times were $10.37 \pm 0.27 \mathrm{~s}$ (range: $9.95-10.63 \mathrm{~s}$ ). After a standardized $45-\mathrm{min}$ warm-up, subjects performed seven maximal sprints in an indoor stadium $(2 \times 10,2 \times 15,20,30$ and $40 \mathrm{~m}$ with 4 -min rest between each trial) in order to collect GRF data over an entire 40-m distance. From these sprints, antero-posterior and vertical GRF components; $\mathrm{F}-\mathrm{v}, \mathrm{P}-\mathrm{v}$, and $R F-\mathrm{v}$ relationships; and associated variables $\left(F_{0}, v_{0}, P_{\max }, S_{\mathrm{Fv}}, D_{\mathrm{RF}}\right)$ were obtained from both force plate measurements and above-described computation method.

\section{Force plate method: materials and data processing}

The experimental protocol used here to determine $\mathrm{F}-\mathrm{v}$ and $\mathrm{P}-\mathrm{v}$ relationships from force plate measurements has recently been proposed and detailed by Rabita et al. (2015). Briefly, during seven sprints, vertical and antero-posterior GRF components were measured by a $6.60-\mathrm{m}$ long force platform system (natural frequency $\geq 500 \mathrm{~Hz}$ ). This system consisted of six individual force plates $(1.2 \times 0.6 \mathrm{~m})$ connected in series, time synchronized, and covered with a tartan mat leveled with the stadium track. Each

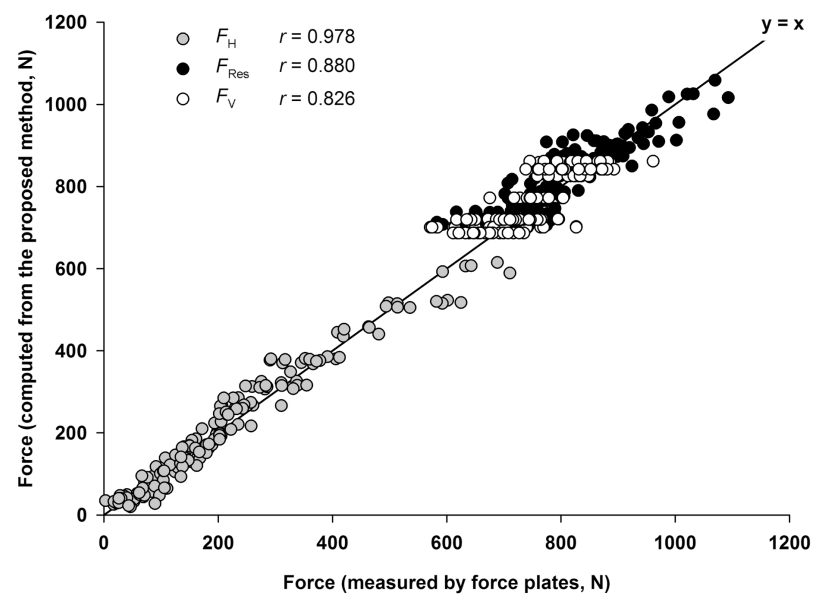

Fig. 1. Correlation between force values measured at each step using the force plate method and values computed by the proposed method. Horizontal and vertical components and resultant ground reaction force values are represented for all subjects. The identity line is represented by the continuous black line.

force plate was equipped with piezoelectric sensors (KI 9067; Kistler, Winterthur, Switzerland). The force signals were digitized at a $1000-\mathrm{Hz}$ sampling rate.

The protocol was designed in order to virtually reconstruct for each athlete the GRF signal of an entire single 40-m sprint by setting differently for each sprint the position of the starting blocks relatively to the $6.60-\mathrm{m}$ long force platform system. The starting blocks were placed over the first platform for the 10-m sprints and were placed remotely for the other trials $(15-40 \mathrm{~m})$ so that 17 different steps (18 foot contacts) from the block to the 40-m mark could be measured (cf. Fig. 1 in Rabita et al., 2015). Force platform signal was low-pass filtered (200-Hz cutoff, third-order zerophase Butterworth) and instantaneous data of vertical $\left(F_{\mathrm{V}}\right.$, in $\left.\mathrm{N}\right)$ and horizontal antero-posterior $\left(F_{\mathrm{H}}\right.$, in $\left.\mathrm{N}\right)$ GRF components as well as the resultant $\left(F_{\text {Res }}\right)$ were averaged for each step consisting of a contact and an aerial phase (determined using 10-N threshold on $F_{\mathrm{V}}$ signal).

Over each measurement areas, the instantaneous horizontal velocity of the $\mathrm{CM}\left(v_{\mathrm{H}}\right.$, in $\left.\mathrm{m} / \mathrm{s}\right)$ was computed as the first integration over time of the antero-posterior horizontal acceleration $\left(a_{\mathrm{H}}\right.$, in $\mathrm{m} / \mathrm{s}^{2}$ ) obtained at each instant dividing $F_{\mathrm{H}}$ by body mass, with initial $v_{\mathrm{H}}$ values as integration constants obtained as follows. For the $10-\mathrm{m}$ sprints, the initial $v_{\mathrm{H}}$ was set to 0 as the starting blocks were placed over the force plate area. For the other sprints, the initial $v_{\mathrm{H}}$ values were measured by high-speed video using a 300 frames per second digital camera (Exilim EX-F1, Casio, Tokyo, Japan) placed perpendicularly to the sagittal plane of motion of the athletes in a fixed position focusing on the entrance of the force plate area (for more details, see Rabita et al., 2015). The instantaneous power output in the horizontal direction $\left(P_{\mathrm{H}}\right)$ was computed as the product at each instant of $F_{\mathrm{H}}$ and $v_{\mathrm{H}}$. Instantaneous data of $v_{\mathrm{H}}$ and $P_{\mathrm{H}}$ were averaged over each step. For the following analyses, the data of the seven sprints were pooled to reconstruct a complete 40-m dataset for each subject. For each step, $R F$ was obtained from averaged step values of $F_{\mathrm{H}}$ and $F_{\text {Res }}$ using eqn. [12]. Individual $D_{\mathrm{RF}}$ values were determined as the slope of the linear $R F-v$ relationships.

\section{Proposed computation method: material and specific data processing}

Sprint times were measured with a pair of photocells (Microgate, Bolzano, Italy) located at the finish line of the seven sprints. For 
each sprint, the timer was triggered when subjects' right thumb left the ground. To remove all possible bias due to this kind of triggering procedure on variables obtained with this computation method, the time between the beginning of the force production on the starting blocks (which represents the actual start of the sprint) and the trigger of the timer was determined and added to photocell times using sagittal high-speed video recording of sprint starts (Exilim EX-F1, Casio, Tokyo, Japan) synchronized with force plate data.

For the two 10- and 15-m trials, only the best times were considered at each distance for data analysis. For each subject, the five split times at $10,15,20,30$, and $40 \mathrm{~m}$ were then used to determine $v_{H_{\max }}$ and $\tau$ using eqn. [3] and least-square regression method. From these two parameters, $v_{\mathrm{H}}(t)$ and $a_{\mathrm{H}}(t)$ were modeled over time using eqns. [1] and [5], respectively. From $a_{\mathrm{H}}(t), F_{\mathrm{H}}(t)$ was modeled over time using eqn. [6] and estimation of $F_{\text {aero }}(t)$ from eqns. [7] to [10]. For individual computations of $k$, both the subject's body mass and stature were measured before tests, $\mathrm{Pb}$ was 760 Torr, and $\mathrm{T}^{\circ}$ was $20^{\circ} \mathrm{C}$. No wind was present during this indoor testing session and the very limited effect of air humidity on $\rho$ was not considered. $P_{\mathrm{H}}$ can then be modeled at each instant as the product of $F_{\mathrm{H}}$ and $v_{\mathrm{H}} . F_{\mathrm{V}}(t)$ and $R F(t)$ were obtained using eqns. [11] and [12], respectively, and individual $D_{\mathrm{RF}}$ values were determined as described in the theoretical background section. All these variables were computed every $0.1 \mathrm{~s}$ (from eqns. [1], [6], [11], and [12]) over each individual acceleration phase.

\section{Common data analysis for both methods}

Individual $\mathrm{F}-\mathrm{v}$ and $\mathrm{P}-\mathrm{v}$ relationships were determined for both methods from step averaged (for force plate method) and modeled (for proposed method) $F_{\mathrm{H}}, P_{\mathrm{H}}$, and $v_{\mathrm{H}}$ values using least-square linear and second-order polynomial regressions, respectively (Jaskolska et al., 1999b; Morin et al., 2010, 2012; Rabita et al., 2015). F-v relationships were extrapolated to obtain $F_{0}$ and $v_{0}$ as the intercepts of the F-v curve with the force and velocity axis, respectively. $S_{\mathrm{Fv}}$ value was determined for each subject as the slope of the F-v relationship, and $P_{\max }$ was determined as the apex of the $\mathrm{P}-\mathrm{v}$ relationship using the first mathematical derivation of the associated quadratic equation. $P_{\max }$ values were also computed as previously proposed and validated (Vandewalle et al., 1987; Samozino et al., 2012, 2014) as follows:

$$
P_{\max }=\frac{F_{0} \cdot v_{0}}{4}
$$

\section{Statistical analysis}

All data are presented as mean \pm standard deviation (SD). For each subject, the standard errors of estimate (SEE) of $F_{\mathrm{H}}, F_{\mathrm{V}}, F_{\text {Res }}$, and $R F$ were computed between values obtained from force plate data at each step and values estimated from model computations for corresponding $v_{\mathrm{H}}$ values:

$$
S E E=\sqrt{\frac{\sum\left(F_{\text {Force Plate }}-F_{\text {Model }}\right)^{2}}{N_{\text {steps }}-2}}
$$

The correlation between force values $\left(F_{\mathrm{H}}, F_{\mathrm{V}}, F_{\mathrm{Res}}\right)$ obtained by both methods was analyzed. F-v relationships, power output capabilities, and mechanical effectiveness obtained with both methods were compared through $F_{0}, v_{0}, S_{\mathrm{FV}}, P_{\max }$, and $D_{\mathrm{RF}}$ values using bias and limits of agreements (Bland \& Altman, 1986). To complete this quantification of inter-method differences, absolute bias was also calculated for each subject as follows: absolute bias $=\mid$ (computation method-force plate method)/force plate methodl.100. For all statistical analyses, a $P$-value of 0.05 was accepted as the level of significance.

\section{Second experimental protocol: inter-trial reliability}

Six high-level sprinters (age: $22.5 \pm 3.9$ years; body mass: $81.8 \pm 5.1 \mathrm{~kg}$; height: $1.86 \pm 0.04 \mathrm{~m}$ ) performed three maximal 50 -m sprints using starting blocks with $10 \mathrm{~min}$ of rest between each trial. Instantaneous $v_{\mathrm{H}}$ was measured at a sampling rate of $46.875 \mathrm{~Hz}$ with a radar system (Stalker ATS System, Radar Sales, Minneapolis, Minnesota, USA) placed on a tripod $10 \mathrm{~m}$ behind the subjects at a height of $1 \mathrm{~m}$ corresponding approximately to the height of subjects' CM (di Prampero et al., 2005; Morin et al., 2012). $F_{0}, v_{0}, S_{\mathrm{FV}}, P_{\max }$, and $D_{\mathrm{RF}}$ values were obtained with the same data processing as presented before for the proposed computation method, except that $v_{H_{\max }}$ and $\tau$ were determined from $v_{\mathrm{H}}(t)$ using eqn. [1] and least-square regression method. Because only the best of several trials is commonly used to be considered during explosive performance testing, the inter-trial reliability of each variable was quantified by the coefficient of variation $(C V$ in $\%$ ), the change in the mean, and the standard error of measurement (SEM, expressed in percentage of mean values) between the two best trials (Hopkins, 2000). These data were used to determine the smallest worthwhile change (SWC) for intra-individual $\left(S W C_{\text {intra }}\right)$ and for inter-individual $\left(S W C_{\text {inter }}\right)$ comparisons for each variable as 0.3 of the SEM (expressed in the variable unity) and 0.2 of the between-subject SDs, respectively (Hopkins, 2004).

\section{Results}

For the validation protocol, the split times at 10, 15, 20, 30 , and $40 \mathrm{~m}$ were $1.84 \pm 0.10,2.49 \pm 0.11,3.05 \pm 0.13$, $4.08 \pm 0.18$, and $5.10 \pm 0.25 \mathrm{~s}$, respectively. For each subject, the change in horizontal position with time given by these split times was well fitted by the exponential model described by eqn. [3] $\left(r^{2}>0.999\right.$; $P<0.0001)$. The associated $v_{H_{\max }}$ and $\tau$ were $10.05 \pm 0.66 \mathrm{~m} / \mathrm{s}$ and $1.24 \pm 0.14 \mathrm{~s}$, respectively. The modeled values of $F_{\mathrm{H}}, F_{\mathrm{V}}, F_{\text {Res }}$, and $P_{\mathrm{H}}$ well fitted the experimental values measured at each step using force plates, which is shown by SEE of $39.8 \pm 13.3 \mathrm{~N}$, $49.5 \pm 17.0 \mathrm{~N}, \quad 52.8 \pm 16.5 \mathrm{~N}, \quad$ and $234.4 \pm 69.9 \mathrm{~W}$, respectively. Force values measured by force plate method at each step and force values computed for the corresponding step using the proposed method were highly correlated $(P<0.001 ;$ Fig. 1$)$. Figure 2 shows the changes in step-averaged (force plate method) and modeled (computation method) values of force, power output, velocity, and RF over the acceleration phase obtained for a typical subject (subject \#5). The SEE of $R F$ over the acceleration phase was $3.72 \% \pm 0.76 \%$.

For all subjects and considering both methods, $\mathrm{F}-\mathrm{v}$ relationships were well fitted by linear regressions (median $r^{2}=0.953$ from 0.920 to 0.987 for the force plate method and $r^{2}>0.999$ for the proposed simple method, $P<0.001)$, $\mathrm{P}-\mathrm{v}$ relationships were well fitted by second-order polynomial regressions (median $r^{2}=0.886$ from 0.857 to 0.961 for the force plate method and $r^{2}=1.000$ for the proposed simple method, $P<0.001$ ), and $R F-v$ relationships were well fitted by linear regressions (median $r^{2}=0.965$ from 0.955 to 

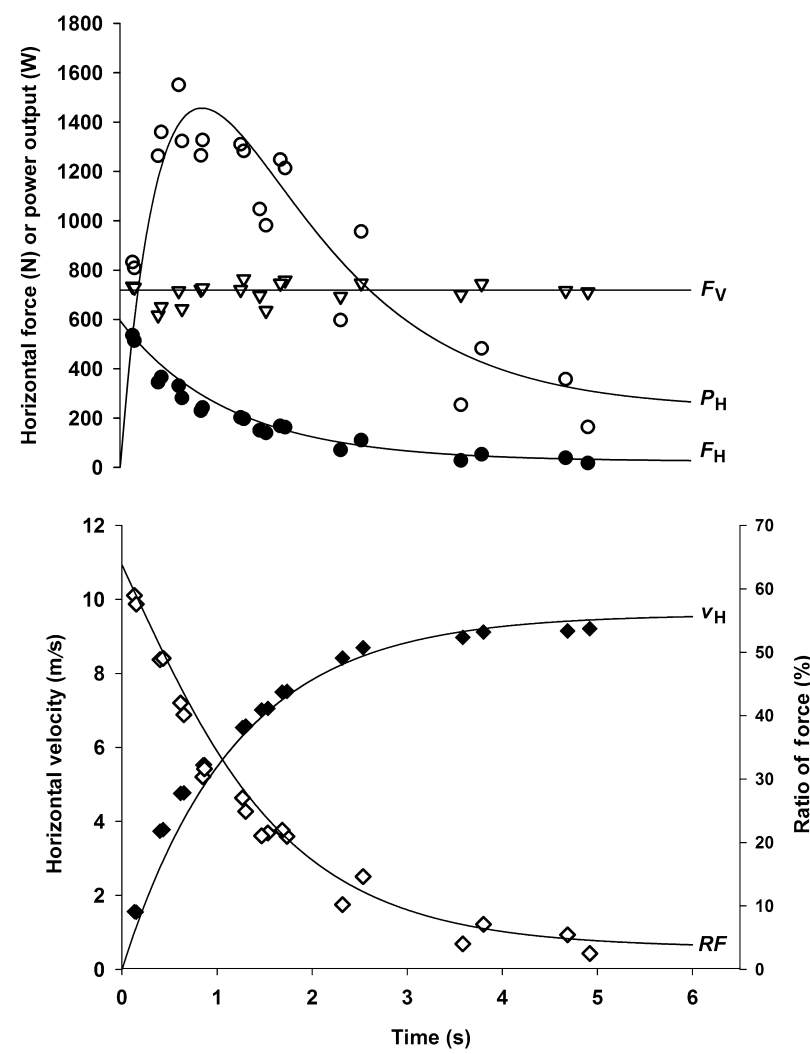

Fig. 2. Changes over the acceleration phase in horizontal velocity $\left(v_{\mathrm{H}}\right.$, black diamonds), horizontal antero-posterior $\left(F_{\mathrm{H}}\right.$, black filled circles) and vertical ( $F_{\mathrm{V}}$, open triangles) force components, horizontal antero-posterior power output ( $P_{\mathrm{H}}$, open circles), and ratio of force ( $R F$, open diamonds) for a typical subject (subject \#5). Points represent averaged values over each step obtained from force plate method (from five sprints) and lines represent modeled values computed by the proposed simple method.

0.976 for the force plate method and $r^{2}>0.996$ for the proposed simple method, $P<0.001)$. Typical examples of these relationships obtained by both methods are shown in Fig. 3 for subject \#5. Values of $F_{0}, v_{0}, P_{\max }$ (both computed as the apex of the P-v relationship and using eqn. [13]), $S_{\mathrm{FV}}$, and $D_{\mathrm{RF}}$ are presented in Table 1 , as well as the associated bias, 95\% limits of agreement, and absolute bias between both methods. Table 2 presents the inter-trial reliability of different mechanical variables through $C V$, change in the mean and SEM between the two best trials of the reliability protocol, as well as values of $S W C_{\text {intra }}$ and $S W C_{\text {inter. }}$.

\section{Discussion}

Although running mechanics over the entire sprint acceleration phase have very recently been described for the first time in overground conditions using in-serie force plates (Rabita et al., 2015), the present study showed valid estimates of the main sprinting mechanical variables from only basic anthropometric and spatiotemporal data (i.e., distance-time or speed-time measurements). The proposed simple computation method
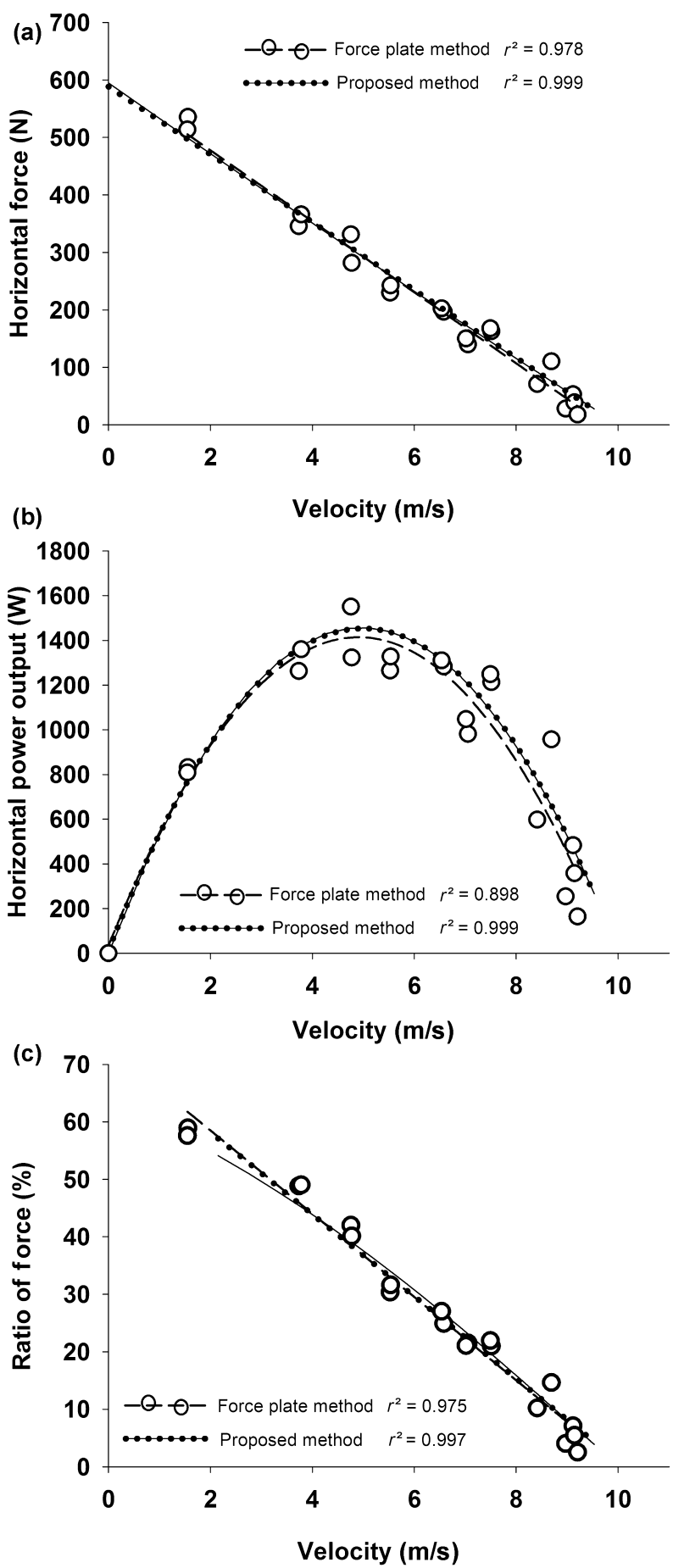

Fig. 3. Force-velocity (a), power-velocity (b), and RF-velocity (c) relationships obtained by both methods for a typical subject (subject \#5). Open circles represent averaged values over each step obtained from force plate method, dashed lines the associated regressions, and thin lines the modeled values computed by the proposed simple method confounded with the associated regressions (dotted lines).

makes possible to determine force- and power-velocity relationships and effectiveness of force application during sprint running acceleration in real-practice conditions. This new method shows very strong agreement with the gold standard force plate measurements and a 
Table 1. Mean \pm SD values of variables attesting the concurrent validity of the proposed method

\begin{tabular}{|c|c|c|c|c|c|}
\hline & $\begin{array}{l}\text { Reference } \\
\text { method }\end{array}$ & $\begin{array}{l}\text { Proposed } \\
\text { method }\end{array}$ & Bias & $\begin{array}{l}95 \% \text { agreement } \\
\text { limits }\end{array}$ & $\begin{array}{l}\text { Absolute } \\
\text { bias (\%) }\end{array}$ \\
\hline$F_{0}(\mathrm{~N})$ & $654 \pm 80$ & $638 \pm 84$ & $-15.9 \pm 25.7$ & $(-66.3 ; 34.5)$ & $3.74 \pm 2.69$ \\
\hline$V_{0}(\mathrm{~m} / \mathrm{s})$ & $10.20 \pm 0.36$ & $10.51 \pm 0.74$ & $0.32 \pm 0.52$ & $(-0.7 ; 1.3)$ & $4.77 \pm 3.26$ \\
\hline$P_{\max }(\mathrm{W})$ apex of the $P-V$ relationship & $1546 \pm 195$ & $1661 \pm 277$ & $115 \pm 107$ & $(-94.7 ; 324.7)$ & $8.04 \pm 5.01$ \\
\hline$P_{\max }(\mathrm{W})$ from $F_{0}$ and $v_{0}$ (eqn. [13]) & $1669 \pm 253$ & $1680 \pm 280$ & $10.56 \pm 45.01$ & $(-77.7 ; 98.8)$ & $1.88 \pm 1.88$ \\
\hline$S_{\mathrm{FV}}(\mathrm{N} / \mathrm{s} / \mathrm{m})$ & $-64.06 \pm 6.30$ & $-60.8 \pm 7.71$ & $3.26 \pm 5.22$ & $(-6.97 ; 13.49)$ & $7.93 \pm 5.32$ \\
\hline$D_{\mathrm{RF}}(\% / \mathrm{s} / \mathrm{m})$ & $-6.80 \pm 0.28$ & $-6.80 \pm 0.74$ & $-0.002 \pm 0.58$ & $(-1.139 ; 1.135)$ & $6.04 \pm 5.70$ \\
\hline
\end{tabular}

SD, standard deviation.

Table 2. Mean \pm SD of the main variables attesting the reliability of the proposed method

\begin{tabular}{|c|c|c|c|c|c|}
\hline & $C V(\%)$ & $\begin{array}{l}\text { Change in } \\
\text { the mean }\end{array}$ & $\begin{array}{l}\text { Standard error of } \\
\text { measurement }(\%)\end{array}$ & $S W C_{\text {intra }}$ & $S W C_{\text {inter }}$ \\
\hline$F_{0}(\mathrm{~N})$ & $2.93 \pm 2.00$ & $-1.53 \pm 32.2$ & 3.57 & 6.76 & 12.06 \\
\hline$v_{0}(\mathrm{~m} / \mathrm{s})$ & $1.11 \pm 0.86$ & $-0.171 \pm 0.776$ & 1.40 & 0.166 & 0.268 \\
\hline$P_{\max }(\mathrm{W})$ apex of the $P-V$ relationship & $1.90 \pm 1.40$ & $-0.164 \pm 0.669$ & 2.37 & 0.147 & 0.441 \\
\hline $\begin{array}{l}P_{\max }(\mathrm{W}) \text { from } F_{0} \text { and } v_{0}(\text { eqn. [13]) } \\
1.87 \pm 1.36\end{array}$ & $1.87 \pm 1.36$ & $-0.167 \pm 0.66$ & 2.33 & 0.144 & 0.446 \\
\hline$S_{\mathrm{FV}}(\mathrm{N} / \mathrm{s} / \mathrm{m})$ & $4.04 \pm 2.72$ & $-0.20 \pm 4.18$ & 4.94 & 0.888 & 0.963 \\
\hline$D_{\mathrm{RF}}(\% / \mathrm{s} / \mathrm{m})$ & $3.99 \pm 2.80$ & $-0.110 \pm 0.45$ & 4.86 & 0.096 & 0.080 \\
\hline
\end{tabular}

SD, standard deviation; SWC, smallest worthwhile change.

high test-retest reliability, which supports its interest for practitioners in numerous sports that involve sprint running accelerations.

\section{Modeled GRFs during sprint acceleration}

The proposed method is based on a macroscopic biomechanical model using an inverse dynamic approach applied to the runner body $\mathrm{CM}$ during sprint running acceleration. This approach models the horizontal antero-posterior and vertical GRF components applied to the runner's CM, and in turn the force developed by the runner onto the ground in the sagittal plane, during the entire acceleration phase of maximal intensity sprint. The main simplifying assumptions admitted in this model are those inherent to the application of fundamental laws of dynamics to the whole human body considered as a system represented by its CM (e.g., Cavagna et al., 1971; van Ingen Schenau et al., 1991; Samozino et al., 2008; Helene \& Yamashita, 2010; Samozino et al., 2010; Samozino et al., 2012; Rabita et al., 2015), the estimation of the horizontal aerodynamic drag from only stature, body mass and a fixed drag coefficient (Arsac \& Locatelli, 2002), and the assumption of a quasi-null CM vertical acceleration over the acceleration phase of the sprint. Note that our computations lead to modeled values over complete steps, i.e., contact plus aerial times, in contrast with previous kinetic measurements during sprint running that averaged mechanical variables over each support phase (Morin et al., 2010, 2011a, 2012; Lockie et al., 2013; Kawamori et al., 2014; Rabita et al., 2015). This induces lower values of force or power output and a different averaging period than support phase-averaged values: step-averaged variables characterize more the mechanics of the overall sprint running propulsion than specifically the mechanical capabilities of lower limb neuromuscular system during each contact phase. This does not affect $R F$ (and in turn $D_{\mathrm{RF}}$ ) values as it is a ratio between two force components averaged over the same duration.

Despite the above-described simplifying assumptions, present results showed that the modeled force values $\left(F_{\mathrm{H}}\right.$, $\left.F_{\mathrm{V}}, F_{\text {Res }}\right)$ were very close to values measured by force plates at each step with low SEE values of $\sim 30-50 \mathrm{~N}$. Furthermore, the values obtained by both methods were highly correlated and closely distributed around the identity line, even if the correlation was slightly lower for $F_{\mathrm{V}}$ than for $F_{\mathrm{H}}$ denoting a better accuracy of estimation in the horizontal than in the vertical direction. It is worth noting that the SEE quantified here are mainly due to the relatively high inter-step variability measured by force plates rather than an inaccuracy of the proposed method. Indeed, as shown in Fig. 2 for a typical subject, force-modeled values well fitted the force plate data with a scattering of the latter due to a possible asymmetry between right and left legs, an inter-step variability inherent to the complexity of this multijoint free movement characterized by a great muscle coordination complexity, and the fact that force plate data were obtained from five different sprints. The inter-step variability is not detectable by the proposed method as the model gives the average tendency of change in GRF components with time. The inter-step variability in force plate data is more obvious in power output values combining the variability of both force and velocity values, which lead to a relatively higher, but still very acceptable, 
power output SEE ( $234 \mathrm{~W})$. For all subjects, $F_{\mathrm{V}}$ values measured with force plates over the first 20-30 m were not particularly higher than body weight (as shown for a typical subject in Fig. 2) and were very close to body weight when averaged over the entire $40 \mathrm{~m}$ (grand averaged difference between mean $F_{\mathrm{V}}$ and body weight of $-2.40 \%$ ). This supports the assumption of a quasi-null vertical acceleration of the $\mathrm{CM}$ over this phase due to a very smoothed upward movement, and in turn supports the validity of step averaged $F_{\mathrm{V}}$ modeled values as equal to body weight. Collectively, these results support a very good agreement in the model determination of GRF in the sagittal plane of motion (horizontal, vertical, and resultant) during sprint running acceleration.

\section{Validity of force- and power-velocity}

relationships determination

Both methods presented individual $\mathrm{F}-\mathrm{v}$ relationships strongly described by linear regression $\left(r^{2}>0.920\right)$ as reported in our recent paper (Rabita et al., 2015), during treadmill sprint running protocols (Jaskolska et al., 1999b; Morin et al., 2010, 2012) or more generally during multijoint lower limb movements such as pedaling, squat, or leg press movements (e.g., Yamauchi \& Ishii, 2007; Dorel et al., 2010; Bobbert, 2012; Samozino et al., 2012). The adjustment quality of the linear regressions was logically better for the proposed method (mean $r^{2}=1.00$ ) based on modeled values than for the reference method (mean $r^{2}=0.956$ ) using experimental force plate data that were inevitably associated to a higher measurement and inter-step variability. The low bias associated to narrow $95 \%$ agreement limits crossing 0 in the determination of $F_{0}, v_{0}$, and $S_{\mathrm{FV}}$ showed that the difference between both methods is very low $(-2.43 \%$, $3.14 \%$, and $5.09 \%$ when expressed relatively to reference values, respectively) and can be attributed to measurements variability. The very low absolute bias $(<5 \%)$, representing exactly the mean absolute error value between both methods at each $\mathrm{F}-\mathrm{v}$ variable determination, clearly supports the validity of the proposed method to determine $\mathrm{F}-\mathrm{v}$ relationship in sprint running. Note that the absolute bias for $S_{\mathrm{FV}}$ was slightly higher than for $F_{0}$ and $v_{0}$ as it represents a regression slope (often associated to higher variability than other variables) computed from $F_{0}$ and $v_{0}$, thus including twice variability.

Individual $\mathrm{P}-\mathrm{v}$ relationships presented well-fitted quadratic regressions for both methods, as expected from previous sprint running protocols (Jaskolska et al., 1999b; Morin et al., 2010, 2012; Rabita et al., 2015). However, for force plate method, the adjustment quality of $\mathrm{P}-\mathrm{v}$ regressions was lower (mean $r^{2}=0.894$ ) than that of $\mathrm{F}-\mathrm{v}$ relationships, and lower than for the proposed method (mean $r^{2}=1.00$ ). This can be explained by the above-discussed higher inter-step and inter-sprint variability measured by force plate and by the very few number of steps in the ascending part of the $\mathrm{P}-\mathrm{v}$ relationship inherent to maximal power production occurring after only approximately five to six steps (Rabita et al., 2015). The lower adjustment quality of the quadratic model obtained from force plate data induced noise in the determination of $P_{\max }$ from the apex of $\mathrm{P}-\mathrm{v}$ relationships, which explains the higher, but still very acceptable, bias and absolute bias $(\sim 8 \%)$ observed here between both methods for this variable compared with bias observed for $F_{0}$ and $v_{0}$. Consequently, differences in $P_{\max }$ are mainly due to the inter-step variability detected by force plates, which was supported by the very similar $P_{\max }$ values obtained using the proposed method from the apex of the $\mathrm{P}-\mathrm{v}$ relationships and using eqn. [13] ( 1\%), while they were quite different for the reference method $(\sim 8 \%)$. From a purely mathematical point of view, if the $\mathrm{F}-\mathrm{v}$ relationship is perfectly linear, the apex of the $\mathrm{P}-\mathrm{v}$ relationships should be equal to $P_{\max }$ given by eqn. [13] (Vandewalle et al., 1987; Samozino et al., 2012). Moreover, when computed from eqn. [13], $P_{\max }$ values obtained by both methods were very close with very low bias values (absolute bias $<2 \%$ ) and narrowed limits of agreement. These findings support the validity of the proposed method in determining $\mathrm{F}-\mathrm{v}$ and $\mathrm{P}-\mathrm{v}$ relationships and their associated mechanical variables $\left(P_{\max }, F_{0}\right.$, $\left.v_{0}, S_{\mathrm{FV}}\right)$ in sprint running.

\section{Validity of the effectiveness of force application determination}

The very good agreement between both methods in GRF components resulted in modeled $R F$ values similar to those computed at each step from force plate measurements, as shown by the low SEE. $R F$ values measured here from both methods (RF values between $0 \%$ and $60 \%$ ) were in line with values previously reported from dynamometric treadmill measurements (between 10\% and 40\%; Morin et al., 2011a, b; Morin et al., 2012), but through a larger range of values as treadmill running made impossible to measure $R F$ for the first step (due to the starting crouch position) and forced subjects to overcome treadmill belt bed horizontal friction force at peak running velocity, the latter being $\sim 20 \%$ lower than the overground one (Morin \& Seve, 2011). Individual $R F-$ velocity relationships determined by both methods were well fitted by inverse linear regressions $\left(r^{2}>0.95\right)$, as originally shown on treadmill (Morin et al., 2011a, 2012) and more recently during overground sprint running (Rabita et al., 2015). Moreover, present results show that the rate of decrease of these regressions $\left(D_{\mathrm{RF}}\right)$ were very similar between both methods (absolute bias $\sim 6 \%$ ). This supports the accuracy of the proposed method to determine the effectiveness of force application throughout the acceleration phase of a sprint and, in turn, its rate of decrease when running velocity increases. 


\section{Validation protocol}

The validation of the proposed method was performed using a recent validated multiple sprint protocol (Rabita et al., 2015) as the reference method. The input variables of the present model are basic anthropometric (body mass and stature) and spatiotemporal data: split times (as used here) or running velocity measurements (as could be obtained from radar guns, e.g., di Prampero et al., 2005; Morin et al., 2006, or laser beams, e.g., Bezodis et al., 2012) during one single sprint. Because the aim of the present study was to validate the whole approach and the proposed equations, using split times from several sprints allowed us to compare exactly the same exercises between the two methods. Using radar data would have forced us to compare mechanical variables obtained from one single sprint using the proposed method to data obtained from five sprints with the reference method, which could have added a bias that would have only been associated to the validation protocol itself. However, the proposed method was also tested in the present protocol from data measured using a radar (Stalker ATS System, Radar Sales, $46.875 \mathrm{~Hz}$ ) during the best sprints of the 30 and $40-\mathrm{m}$ trials. Results were very similar to those obtained from split times, with slightly higher bias values (absolute bias from 3\% to 7\%) due to the abovediscussed point, which supports the validity of the proposed method from running velocity measurements. As partly noticed by Furusawa et al. (1927), the present macroscopic biomechanical model shows that, whatever the kind of locomotion, when displacement velocity changes with time follow an exponential function (as described by eqn. [1] and previously reported during maximal sprint acceleration from recreational to highly trained sprinters; di Prampero et al., 2005; Morin \& Seve, 2011; Morin et al., 2012), the relationship between horizontal GRF component and velocity is quasi-linear.

\section{Reliability}

The reliability of the proposed method was tested here in a second protocol as the first mentioned protocol already required seven maximal sprints to compare the proposed to the reference method. For all the mechanical variables, low $C V$ and $S E M$ values $(<5 \%)$ associated to change in the mean close to 0 showed the high Test-retest reliability of the proposed method. The present level of reliability is in accordance with reliability previously reported during isoinertial all out tests (e.g., Hopkins et al., 2001; Samozino et al., 2008). This high reliability led to low $S W C$ values for both intra- and interindividual comparisons for each variable and strongly that this simple method is of great interest for sport practitioners and clinicians to detect training or rehabilitation effects, or for scientists when using progressive magnitude statistics.

\section{Practical applications}

In light of the above-discussed points, the present study proposed an accurate, reliable, valid, and simple method to determine $\mathrm{F}-\mathrm{v}, \mathrm{P}-\mathrm{v}$, and $R F-\mathrm{v}$ relationships during overground sprint running from variables easily obtained in field conditions and with a precision similar to that obtained with specific laboratory devices (force plates; Rabita et al., 2015). This new field method is based on the previously validated simple method focusing on the same kind of mechanical properties during jumping movements and based on a similar biomechanical macroscopic approach (Samozino et al., 2008, 2014). The present proposed method only requires individual basic anthropometric data (body mass and stature) and $\sim 5$ split times or instantaneous velocity measurements obtained during one single sprint acceleration until maximal velocity. Note that split times or instantaneous velocity have become more accessible with new technologies such as position trackers, GPS, or accelerometer-based systems. Anthropometric and split times or instantaneous velocity data can then be used as inputs in the basic data processing to determine $v_{H_{\max }}$ and $\tau$ using least-square regression method from eqns. [1] or [2] and then to compute different mechanical variables (details in the Methods section "Proposed computation method: material and specific data processing" and "Common data analysis for both methods"). This will contribute to generalize such evaluations for both scientific and training purposes as it has the potential to be easily reproduced by coaches, sport practitioners, or physiotherapists in their daily practice. Such a testing session could take only 15-20 min after a regular warm-up with two or three sprints by athlete, only the best one being analyzed using the method proposed here.

The variables obtained from $\mathrm{F}-\mathrm{v}, \mathrm{P}-\mathrm{v}$, and $R F-\mathrm{v}$ relationships give key information about force, velocity, and power output capabilities, and about the effectiveness of force application, which are of great interest to optimize sprint running acceleration performance by comparing $\mathrm{P}-\mathrm{F}-\mathrm{v}$ qualities of different athletes, orienting and individualizing training loads exercises, and monitoring training or rehabilitation in sports using sprint accelerations (e.g., track-and-field events, team sports). Indeed, as previously mentioned, sprint performance has been shown to be related to these mechanical variables, notably $P_{\max }, v_{0}$, and $D_{\mathrm{RF}}$ (Morin et al., 2011a, 2012; Rabita et al., 2015). Furthermore, these mechanical variables seem to be sensitive to training modalities (notably through in-season variations, unpublished personal data). For instance, using weighted sled towing improves horizontal force production and force application effectiveness in sprint running (Cronin et al., 2008; Kawamori et al., 2014). Note that methods using force plate system with several sprints or instrumented sprint treadmill would give the same kind of information, but are currently impossible to set in training practice for 
most sport practitioners. That said, they are still very interesting for studying inter-step variability or for intrastep analyses, contact and aerial times, step length/ frequency and force impulse, and rate of development during sprint running, or for other analyses requiring additional and synchronized laboratory measurements.

\section{Perspectives}

This study proposed an accurate, reliable, and valid simple method to evaluate mechanical properties of overground sprint running propulsion and validated it through a very good agreement with gold standard force plate measurements. The proposed method is based on a macroscopic biomechanical model using an inverse dynamic approach applied to the runner's CM. This method is convenient for field use by sport practitioners and clinicians as it requires only anthropometric (body mass and stature) and spatiotemporal (split times or instantaneous velocity) variables easy to obtain out of a laboratory during sprint running acceleration. This method could be further used to increase the understand- ing in the mechanical determinants of sprint acceleration performance in many sports, to study the adaptations of the mechanical properties of lower limb neuromuscular system in a variety of sprint running propulsion, and to optimize sprint performance by individualizing and orienting training or rehabilitation programs.

Key words: Acceleration, maximal performance, evaluation, sprint mechanics, lower limb explosive capabilities, ratio of force application.

\section{Acknowledgements}

We are grateful to Dr Pascal Edouard and Philippe Gimenez (University of Saint-Etienne, France), Pedro Jiménez-Reyes (UCAM, Murcia, Spain), Matt Brughelli (AUT, Auckland, New Zealand), Antoine Couturier, Gaël Guilhem, Caroline Giroux, Stevy Farcy, and Virha Despotova (INSEP, France) for their valuable participation to this project and their enthusiastic and friendly collaboration. We would like to thank Guy Ontanon, Dimitri Demonière, Michel Gilot, Pierre Carraz, and the athletes of both the French Institute of Sport (INSEP) and the Athletic Sport Aixois who voluntarily gave their best performance for this protocol.

\section{References}

Arsac LM, Locatelli E. Modeling the energetics of 100-m running by using speed curves of world champions. J Appl Physiol 2002: 92: 1781-1788.

Bezodis NE, Salo AI, Trewartha G. Measurement error in estimates of sprint velocity from a laser displacement measurement device. Int J Sports Med 2012: 33: 439-444.

Bland JM, Altman DG. Statistical methods for assessing agreement between two methods of clinical measurement. Lancet 1986: 1: 307-310.

Bobbert MF. Why is the force-velocity relationship in leg press tasks quasi-linear rather than hyperbolic? J Appl Physiol 2012: 112: 1975-1983.

Cavagna GA, Komarek L, Mazzoleni S. The mechanics of sprint running. J Physiol 1971: 217: 709-721.

Chelly SM, Denis C. Leg power and hopping stiffness: relationship with sprint running performance. Med Sci Sports Exerc 2001: 33: 326-333.

Cormie P, McGuigan MR, Newton RU. Adaptations in athletic performance after ballistic power vs strength training. Med Sci Sports Exerc 2010a: 42: 1582-1598.

Cormie P, McGuigan MR, Newton RU. Influence of strength on magnitude and mechanisms of adaptation to power training. Med Sci Sports Exerc 2010b: 42: 1566-1581.

Cormie P, McGuigan MR, Newton RU. Developing maximal neuromuscular power: part 1 - biological basis of maximal power production. Sports Med 2011: 41: 17-38.

Cronin J, Sleivert G. Challenges in understanding the influence of maximal power training on improving athletic performance. Sports Med 2005: 35: 213-234.

Cronin J, Hansen K, Kawamori N, McNair P. Effects of weighted vests and sled towing on sprint kinematics. Sports Biomech 2008: 7: 160-172.

di Prampero PE, Fusi S, Sepulcri L, Morin JB, Belli A, Antonutto G. Sprint running: a new energetic approach. J Exp Biol 2005: 208: 2809-2816.

di Prampero PE, Botter A, Osgnach C. The energy cost of sprint running and the role of metabolic power in setting top performances. Eur J Appl Physiol 2015: 115: 451-469.

Dorel S, Couturier A, Lacour JR, Vandewalle H, Hautier C, Hug F. Force-velocity relationship in cycling revisited: benefit of two-dimensional pedal forces analysis. Med Sci Sports Exerc 2010: 42: 1174-1183.

Furusawa K, Hill AV, Parkinson JL. The dynamics of "sprint" running. Proc R Soc Lond B 1927: 102: 29-42.

Helene O, Yamashita MT. The force, power and energy of the 100 meter sprint. Am J Phys 2010: 78: 307-309.

Hill AV. The heat of shortening and the dynamic constants of muscle. Proc $\mathrm{R}$ Soc Lond B Biol Sci 1938: 126B: 136-195.
Hopkins WG. Measures of reliability in sports medicine and science. Sports Med 2000: 30: 1-15.

Hopkins WG. How to interpret changes in an athletic performance test. Sportscience 2004: 8: 1-7.

Hopkins WG, Schabort EJ, Hawley JA. Reliability of power in physical performance tests. Sports Med 2001: 31: 211-234.

Jaskolska A, Goossens P, Veenstra B, Jaskolski A, Skinner JS. Comparison of treadmill and cycle ergometer measurements of force-velocity relationships and power output. Int J Sports Med 1999a: 20: 192-197.

Jaskolska A, Goossens P, Veenstra B, Jaskolski A, Skinner JS. Treadmill measurement of the force-velocity relationship and power output in subjects with different maximal running velocities. Sports Med Train Rehab 1999b: 8: 347-358.

Kawamori N, Newton R, Nosaka K. Effects of weighted sled towing on ground reaction force during the acceleration phase of sprint running. J Sports Sci 2014: 32: 1139-1145.

Lockie RG, Murphy AJ, Schultz AB, Jeffriess MD, Callaghan SJ. Influence of sprint acceleration stance kinetics on velocity and step kinematics in field sport athletes. J Strength Cond Res 2013: 27: 2494-2503.

Morin JB, Seve P. Sprint running performance: comparison between 
treadmill and field conditions. Eur J Appl Physiol 2011: 111: 1695-1703.

Morin JB, Jeannin T, Chevallier B, Belli A. Spring-mass model characteristics during sprint running: correlation with performance and fatigue-induced changes. Int J Sports Med 2006: 27: 158-165.

Morin JB, Samozino P, Bonnefoy R, Edouard P, Belli A. Direct measurement of power during one single sprint on treadmill. J Biomech 2010: 43: 1970-1975.

Morin JB, Edouard P, Samozino P. Technical ability of force application as a determinant factor of sprint performance. Med Sci Sports Exerc 2011a: 43: 1680-1688.

Morin JB, Samozino P, Edouard P, Tomazin K. Effect of fatigue on force production and force application technique during repeated sprints. J Biomech 2011b: 44: 2719-2723.

Morin JB, Bourdin M, Edouard P, Peyrot N, Samozino P, Lacour JR. Mechanical determinants of 100-m sprint running performance. Eur J Appl Physiol 2012: 112: 3921-3930.
Rabita G, Dorel S, Slawinski J, Saez de Villarreal E, Couturier A, Samozino P, Morin JB. Sprint mechanics in world-class athletes: a new insight into the limits of human locomotion. Scand J Med Sci Sports 2015: DOI: 10.1111/sms.12389.

Samozino P, Morin JB, Hintzy F, Belli A. A simple method for measuring force, velocity and power output during squat jump. J Biomech 2008: 41: 2940-2945.

Samozino P, Morin JB, Hintzy F, Belli A. Jumping ability: a theoretical integrative approach. J Theor Biol 2010: 264: 11-18.

Samozino P, Rejc E, Di Prampero PE, Belli A, Morin JB. Optimal force-velocity profile in ballistic movements. Altius: citius or fortius? Med Sci Sports Exerc 2012: 44: 313-322.

Samozino P, Edouard P, Sangnier S, Brughelli M, Gimenez P, Morin JB. Force-velocity profile: imbalance determination and effect on lower limb ballistic performance. Int J Sports Med 2014: 35: 505-510.
Slawinski J, Bonnefoy A, Ontanon G, Leveque JM, Miller C, Riquet A, Cheze L, Dumas R.

Segment-interaction in sprint start: analysis of 3D angular velocity and kinetic energy in elite sprinters. J Biomech 2010: 43: 1494-1502.

Thorstensson A, Grimby G, Karlsson J. Force-velocity relations and fiber composition in human knee extensor muscles. J Appl Physiol 1976: 40: 12-16.

van Ingen Schenau GJ, Jacobs R, de Koning JJ. Can cycle power predict sprint running performance? Eur J Appl Physiol Occup Physiol 1991: 63: 255-260.

Vandewalle H, Peres G, Monod H. Standard anaerobic exercise tests. Sports Med 1987: 4: 268-289.

Yamauchi J, Ishii N. Relations between force-velocity characteristics of the knee-hip extension movement and vertical jump performance. J Strength Cond Res 2007: 21: 703-709. 